Bull. Korean Math. Soc. 48 (2011), No. 6, pp. 1303-1314

http://dx.doi.org/10.4134/BKMS.2011.48.6.1303

\title{
MEROMORPHIC SOLUTIONS OF SOME $q$-DIFFERENCE EQUATIONS
}

\author{
BaoQin Chen and ZongXuan Chen
}

ABSTRACT. We consider meromorphic solutions of $q$-difference equations of the form

$$
\sum_{j=0}^{n} a_{j}(z) f\left(q^{j} z\right)=a_{n+1}(z)
$$

where $a_{0}(z), \ldots, a_{n+1}(z)$ are meromorphic functions, $a_{0}(z) a_{n}(z) \not \equiv 0$ and $q \in \mathbb{C}$ such that $0<|q| \leq 1$. We give a new estimate on the upper bound for the length of the gap in the power series of entire solutions for the case $0<|q|<1$ and $n=2$. Some growth estimates for meromorphic solutions are also given in the cases $0<|q|<1$ and $|q|=1$. Moreover, we investigate zeros and poles of meromorphic solutions for the case $|q|=1$.

\section{Introduction and main results}

Recently many papers (see $[1-4,6-10,13]$ ) focused on complex difference equations and $q$-difference equations. Many meaningful results have been obtained. In this paper, we are concerned about meromorphic solutions of $q$ difference equations of the form

$$
\sum_{j=0}^{n} a_{j}(z) f\left(q^{j} z\right)=a_{n+1}(z),
$$

where $a_{0}(z), \ldots, a_{n+1}(z)$ are meromorphic functions, $a_{0}(z) a_{n}(z) \not \equiv 0$ and $q \in \mathbb{C}$ such that $0<|q| \leq 1$. Throughout this paper, we assume that the reader is familiar with the standard notations and the fundamental results of Nevanlinna theory (see $[11,14,18]$ ). In addition, we use $\rho(f)$ to denote the order of growth of the meromorphic function $f(z)$, and $\lambda(f)$ to denote the exponent of convergence of zeros of $f(z)$. $[3]$.

We firstly recall a result proved by Bergweiler, Ishizaki and Yanagihara in

Received August 14, 2010; Revised January 14, 2011.

2010 Mathematics Subject Classification. Primary 30D35, 39 B32.

Key words and phrases. $q$-difference equation, growth, type.

This project was supported by the National Natural Science Foundation of China (No: 10871076, No: 11171119 and No: 11026096). 
Theorem A ([3]). Let $a_{0}(z), \ldots, a_{n+1}(z)$ be polynomials without common zeros and $0<|q|<1$. Suppose (1.1) possesses a transcendental entire solution $f(z)$. Then there is some $j, 1 \leq j \leq n$, such that $\operatorname{deg} a_{0}(z)<\operatorname{deg} a_{j}(z)$.

In a recent paper [5], we gave estimates on the upper bound for the length of the gap in the power series of entire solutions of (1.1). In fact, we proved the following results.

Theorem B ([5]). Let $0<|q|<1$ and $a_{j}(z)=b_{j} z^{d_{j}}, j=0, \ldots, n$ where $b_{j}$ are constants, $d_{j}$ are nonnegative integer numbers such that $b_{0} b_{n} \neq 0$, and let $a_{n+1}(z)$ be a polynomial. Suppose that (1.1) has a transcendental entire solution

$$
f(z)=\sum_{k=0}^{\infty} \alpha_{v_{k}} z^{v_{k}} \quad\left(\alpha_{v_{k}} \neq 0 \text { are constants }\right) .
$$

Then there is some $k_{0}>0$ such that for any $k>k_{0}$,

$$
v_{k+1}-v_{k} \leq \max _{0 \leq i<j \leq n}\left|d_{j}-d_{i}\right|
$$

Theorem C ([5]). Let $0<|q|<1$ and $a_{0}(z), \ldots, a_{n+1}(z)$ be polynomials. Suppose that (1.1) has a transcendental entire solution denoted by (1.2). Then there is some $k_{0}>0$ such that for any $k>k_{0}$,

$$
v_{k+1}-v_{k} \leq \max \left\{\operatorname{deg} a_{j}: j=0, \ldots, n\right\} .
$$

In what follows, we say $f(z)=\sum_{n=0}^{\infty} \alpha_{\lambda_{n}} z^{\lambda_{n}}\left(\alpha_{\lambda_{n}} \neq 0\right.$ are constants) has a Fabry gap (see [12]), if

$$
\frac{\lambda_{n}}{n} \rightarrow \infty \quad(n \rightarrow \infty)
$$

Corollary D ([5]). Let $0<|q|<1$ and $a_{0}(z), \ldots, a_{n+1}(z)$ be polynomials. Suppose that (1.1) possesses a transcendental entire solution $f(z)$. Then $f(z)$ must not have a Fabry gap.

We improve Theorem $\mathrm{B}$ for the case $n=2$ here by proving the following result.

Theorem 1.1. Let $0<|q|<1$ and $a_{j}(z)=b_{j} z^{d_{j}}, j=0,1,2$, where $b_{j}$ are constants, $d_{j}$ are nonnegative integer numbers such that $b_{0} b_{2} \neq 0$, and let $a_{3}(z)$ be a polynomial such that $a_{3}(0) \neq 0$. Suppose that

$$
a_{2}(z) f\left(q^{2} z\right)+a_{1}(z) f(q z)+a_{0}(z) f(z)=a_{3}(z)
$$

has a transcendental entire solution denoted by (1.2). Then there is some $k_{0}>0$ such that for any $k>k_{0}$,

$$
v_{k+1}-v_{k} \leq \max _{j=1,2}\left|d_{j}-d_{0}\right| .
$$

Next, we recall some results in the case that $0<|q|<1$ and the coefficients $a_{0}(z), \ldots, a_{n+1}(z)$ are not all rational functions. Theorem E below follows from Theorem 3.5 and Corollary 3.6 in [13]. 
Theorem E ([13]). Suppose that the coefficients $a_{0}(z), \ldots, a_{n+1}(z)$ in $(1.1)$ are meromorphic and of finite order $\leq \rho$ and $0<|q|<1$. Then all meromorphic solutions of (1.1) are of finite order $\leq \rho$. In addition, if $\rho\left(a_{n+1}\right)>\rho\left(a_{j}\right)$ for all $j=0, \ldots, n$, then $\rho(f)=\rho\left(a_{n+1}\right)$.

The following two special cases of the coefficients $a_{0}(z), \ldots, a_{n+1}(z)$ were investigated in [5].

Case 1: $a_{j}(z)=P_{j}(z) e^{p_{j} z}+Q_{j}(z), j=0, \ldots, n+1$, where $p_{j}=d_{j} e^{i \theta}, \theta \in$ $[0,2 \pi), P_{j}(z), Q_{j}(z)$ are meromorphic functions with $\max \left\{\rho\left(P_{j}\right), \rho\left(Q_{j}\right)\right\}<1$ and there is some $s \in\{0, \ldots, n+1\}$ such that $d_{s}>\max \left\{d_{j}: j \neq s, 0 \leq j \leq\right.$ $n+1\}$;

Case 2: $a_{j}(z)=P_{j}(z) e^{p_{j} z}+Q_{j}(z) e^{-q_{j} z}+C_{j}(z), j=0, \ldots, n+1$, where $p_{j}$, $q_{j} \geq 0, P_{j}(z), Q_{j}(z), C_{j}(z)$ are meromorphic functions with $\max \left\{\rho\left(P_{j}\right), \rho\left(Q_{j}\right)\right.$, $\left.\rho\left(C_{j}\right)\right\}<1$ and there is some $s \in\{0, \ldots, n+1\}$ such that $P_{s}(z) Q_{s}(z) \not \equiv$ $0, p_{s} q_{s} \neq 0$ while $P_{0}(z)=\cdots=P_{s-1}(z)=P_{s+1}(z)=\cdots=P_{n+1}(z) \equiv 0$, or $Q_{0}(z)=\cdots=Q_{s-1}(z)=Q_{s+1}(z)=\cdots=Q_{n+1}(z) \equiv 0$.

Theorem F ([5]). If $0<|q|<1$ and the coefficients of (1.1) satisfy any one of Cases 1 and 2 listed above, then each non-zero meromorphic solution of (1.1) is of order $\rho(f)=1$.

Some cases similar to Case 1 had been investigated in [13] and some meaningful results were proved. We find that there always exists some dominating coefficient in all these considerations. As a continuation, we give the following result which is a generalization of Theorem $\mathrm{F}$.

Theorem 1.2. Suppose that the coefficients $a_{0}(z), \ldots, a_{n+1}(z)$ in (1.1) are meromorphic and of finite order $\leq \rho$ and $0<|q|<1$. If for any given $\varepsilon>0$, there exist some $l, 0 \leq l \leq n+1$, and an unbounded domain $D \subset \mathbb{C}$, such that for all $z \in D$,

$$
\begin{gathered}
\left|a_{l}(z)\right| \geq \exp \left\{\alpha r^{\rho-\varepsilon}\right\}, \\
\left|a_{j}(z)\right| \leq \exp \left\{\beta r^{\rho-\varepsilon}\right\}, \quad j=0,1, \ldots, l-1, l+1, \ldots, n+1,
\end{gathered}
$$

where $\alpha>\beta>0$ are positive real numbers, then each non-zero meromorphic solution of (1.1) is of order $\rho(f)=\rho$.

To describe the growth of entire solutions more precisely, we consider its type and prove the following results.

Theorem 1.3. Let $0<|q|<1$ and the coefficients $a_{0}(z), \ldots, a_{n+1}(z)$ in $(1.1)$ be entire and of finite order $\leq \rho$ such that among those coefficients having the maximal order $\rho:=\max _{0 \leq j \leq n+1} \rho\left(a_{j}\right)$, exactly one denoted by $a_{l}(z)$ has its type strictly greater than the others, then each non-zero entire solution of (1.1) is of order $\rho(f)=\rho$. Moreover, if $l=n+1$, then we have $\tau(f) \geq$ $\tau\left(a_{n+1}\right)-\max \left\{\tau\left(a_{j}\right): \rho\left(a_{j}\right)=\rho, j \neq n+1\right\}$.

Corollary 1.4. Under the assumptions of Theorem 1.3, if $\tau\left(a_{n+1}\right)=\infty$, then each non-zero entire solution of (1.1) is of order $\rho(f)=\rho$ and of type $\tau(f)=\infty$. 
Example. $f(z)=e^{z}$ satisfies

$$
e^{\frac{1}{4} z} f\left(\frac{1}{4} z\right)-f\left(\frac{1}{2} z\right)+e^{\frac{1}{2} z} f(z)=e^{\frac{3}{2} z} .
$$

This is an example of Theorem 1.3 , where $q=\frac{1}{2}$ and $n=2$. It shows that $\tau(f)=\tau\left(a_{n+1}\right)-\max \left\{\tau\left(a_{j}\right): \rho\left(a_{j}\right)=\rho, j \neq n+1\right\}$ may hold in Theorem 1.3.

We prove Theorem 1.1 and Theorem 1.3 in Section 2 and Section 3 respectively. We omit the proof of Theorem 1.2 as it can be proved with the same idea as in the proof of Theorem 1.3. And we give some results and their proofs for the case $|q|=1$ in Sections $4-6$.

\section{Proof of Theorem 1.1}

Firstly, by (1.2), for each $j \in\{0,1,2\}$, we have

$$
\begin{aligned}
& a_{j}(z) f\left(q^{j} z\right)=a_{j}(z) \sum_{k=0}^{\infty} \alpha_{v_{k}}\left(q^{j} z\right)^{v_{k}}=b_{j} z^{d_{j}} \sum_{k=0}^{\infty} \alpha_{v_{k}}\left(q^{j} z\right)^{v_{k}} \\
& =b_{j} \alpha_{v_{0}} q^{j v_{0}} z^{v_{0}+d_{j}}+b_{j} \alpha_{v_{1}} q^{j v_{1}} z^{v_{1}+d_{j}}+\cdots
\end{aligned}
$$

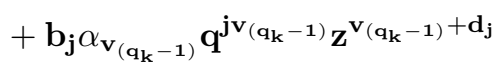

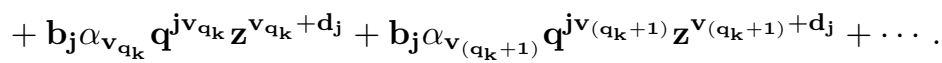

Since $a_{3}(0) \neq 0$, we can see that $a_{j}(z), j=0,1,2,3$, do not have common zeros. By Theorem A, we have $\max \left\{d_{1}, d_{2}\right\}>d_{0}$. What's more, from Theorem B, we see that our conclusion holds for the cases: Case 1. $d_{2}=d_{1}>d_{0}$, Case 2. $d_{2}>d_{1} \geq d_{0}$, and Case 3. $d_{1}>d_{2} \geq d_{0}$. Thus we only need to discuss two cases: Case 4. $d_{2}>d_{0}>d_{1}$ and Case 5. $d_{1}>d_{0}>d_{2}$.

Case 4. $d_{2}>d_{0}>d_{1}$. Suppose that the assertion does not hold, by Theorem $\mathrm{B}$, then for any given $k>0$, there is some $q_{k}>k$ such that

$$
\max \left\{d_{2}-d_{0}, d_{0}-d_{1}\right\}<v_{\left(q_{k}+1\right)}-v_{q_{k}} \leq d_{2}-d_{1} .
$$

If $d_{2}-d_{0}=\max \left\{d_{2}-d_{0}, d_{0}-d_{1}\right\}$, then we can choose an infinitely sequence $\left\{v_{q_{k}}\right\} \subset\left\{v_{k}\right\}$, such that for any $q_{k}$,

$$
v_{\left(q_{k}+1\right)}+d_{1} \leq v_{q_{k}}+d_{2}<v_{\left(q_{k}+1\right)}+d_{0} .
$$

If $v_{\left(q_{k}+1\right)}+d_{1}=v_{q_{k}}+d_{2}$, then we have

$$
\begin{aligned}
& v_{q_{k}}+d_{1}<v_{q_{k}}+d_{0}<v_{q_{k}}+d_{2} \\
= & v_{\left(q_{k}+1\right)}+d_{1}<v_{\left(q_{k}+1\right)}+d_{0}<v_{\left(q_{k}+1\right)}+d_{2} .
\end{aligned}
$$

Substituting (2.1) into (1.3), then arranging the power series of $a_{2}(z) f\left(q^{2} z\right)+$ $a_{1}(z) f(q z)+a_{0}(z) f(z)$ anew in accordance with the monotone nondecreasing degree of $z$, we get that

$$
\begin{aligned}
a_{3}(z) & =a_{2}(z) f\left(q^{2} z\right)+a_{1}(z) f(q z)+a_{0}(z) f(z) \\
& =\cdots+\mathbf{b}_{\mathbf{0}} \alpha_{\mathbf{v}_{\mathbf{q}_{\mathbf{k}}}} \mathbf{z}^{\mathbf{v}_{\mathbf{q}_{\mathbf{k}}}+\mathbf{d}_{\mathbf{o}}}+\cdots .
\end{aligned}
$$


We notice the boldface terms in (2.1) for $j=0,1,2$. By (2.2) and (2.3), we see that in (2.4), there is exactly one term $b_{0} \alpha_{v_{q_{k}}} z^{v_{q_{k}}+d_{0}}$ has degree $v_{q_{k}}+d_{0}$.

For sufficiently large $q_{k}, v_{q_{k}}+d_{0}>\operatorname{deg} a_{3}$, by (2.4), we see that the coefficient of $z^{v_{q_{k}}+d_{0}}$ equals 0 . That is $b_{0} \alpha_{v_{q_{k}}}=0$, a contradiction.

Thus we have $v_{\left(q_{k}+1\right)}+d_{1}<v_{q_{k}}+d_{2}$. Since $d_{2}-d_{0}=\max \left\{d_{2}-d_{0}, d_{0}-d_{1}\right\} \geq$ $d_{0}-d_{1}$, and by $(2.2)$, we obtain

$$
\begin{aligned}
v_{\left(q_{k}+1\right)}+d_{1} & =v_{\left(q_{k}+1\right)}-v_{q_{k}}+v_{q_{k}}+d_{1}>v_{q_{k}}+d_{2}-d_{0}+d_{1} \\
& =v_{q_{k}}+d_{0}+\left(d_{1}+d_{2}-2 d_{0}\right) \geq v_{q_{k}}+d_{0} .
\end{aligned}
$$

Then we have

$$
v_{q_{k}}+d_{1}<v_{q_{k}}+d_{0}<v_{\left(q_{k}+1\right)}+d_{1}<v_{q_{k}}+d_{2}<v_{\left(q_{k}+1\right)}+d_{0} .
$$

Then we can deduce the same contradiction that $b_{0} \alpha_{v_{q_{k}}}=0$ by an argument similar to the above.

If $d_{0}-d_{1}=\max \left\{d_{2}-d_{0}, d_{0}-d_{1}\right\}$, we can deduce the same contradiction without difficulty. This completes the proof of Case 4.

Case 5. $d_{1}>d_{0}>d_{2}$. By reasoning similar to that in the proof of Case 4 , we can prove Case 5 and finish our proof of Theorem 1.1.

\section{Proof of Theorem $\mathbf{1 . 3}$}

Lemma $3.1([15])$. Let $f(z)$ be a meromorphic function with $\rho(f)=\beta<+\infty$. Then for any given $\varepsilon>0$, there exists a set $E \subset(1,+\infty)$ with finite measure, such that for all $z$ satisfying $|z|=r \notin[0,1] \cup E$, and $r$ sufficiently large,

$$
\exp \left\{-r^{\beta+\varepsilon}\right\} \leq|f(z)| \leq \exp \left\{r^{\beta+\varepsilon}\right\} .
$$

Lemma $3.2([16])$. Let $f(z)$ be a nonconstant entire function with $\rho(f)=\rho<$ $\infty, 0<\tau(f)=\tau<\infty$. Then for any given $\beta<\tau$, there exists a set $E \subset(1, \infty)$ with infinite logarithmic measure, such that for all $z$ satisfying $|z|=r \in E$, we have,

$$
\log M(r, f)>\beta r^{\rho} .
$$

Proof of Theorem 1.3. Let $f(z)$ be a non-zero entire solution of (1.1). By Theorem E, we have $\rho(f) \leq \rho$. We next proceed to show that $\rho(f)=\rho$. Suppose that $\rho(f)=\sigma<\rho$, then $\rho\left(\frac{1}{f\left(q^{j} z\right)}\right)=\rho\left(f\left(q^{j} z\right)\right)=\sigma$ for $j=0, \ldots, n$.

Denote $I:=\left\{j \in\{0, \ldots, n+1\} \mid \rho\left(a_{j}\right)=\rho\right\}, \tau:=\max \left\{\tau\left(a_{j}\right): j \in I \backslash\{l\}\right\}$, and $\tilde{\rho}:=\max \left\{\rho\left(a_{j}\right): j \in\{0, \ldots n+1\} \backslash I\right\}<\rho$. From Lemma 3.1, for any given $\varepsilon_{1}\left(0<2 \varepsilon_{1}<\min \{\rho-\sigma, \rho-\tilde{\rho}\}\right)$, there exists a set $E_{1} \subset(1,+\infty)$ with finite measure, such that for all $z=r e^{i \theta}$ satisfying $|z|=r \notin[0,1] \cup E_{1}$, and $r$ sufficiently large, we have

$$
\begin{gathered}
\left|\frac{1}{f\left(q^{l} z\right)}\right| \leq \exp \left\{\left(|q|^{l} r\right)^{\sigma+\varepsilon_{1}}\right\} \leq \exp \left\{r^{\sigma+\varepsilon_{1}}\right\}, \\
\left|f\left(q^{j} z\right)\right| \leq \exp \left\{\left(|q|^{j} r\right)^{\sigma+\varepsilon_{1}}\right\} \leq \exp \left\{r^{\sigma+\varepsilon_{1}}\right\}
\end{gathered}
$$


for each $j \in\{0, \ldots, n+1\} \backslash\{l\}$, and

$$
\left|a_{j}(z)\right| \leq \exp \left\{r^{\tilde{\rho}+\varepsilon_{1}}\right\} \leq \exp \left\{r^{\rho-\varepsilon_{1}}\right\}
$$

for each $j \in\{0, \ldots, n+1\} \backslash I$.

Let $\alpha_{1}, \alpha_{2} \in \mathbb{R}$ be two constants such that $\tau<\alpha_{1}<\alpha_{2}<\tau\left(a_{l}\right)$. By Lemma 3.2 and the definition of the type of an entire function, we see that there exists a set $E_{2} \subset(1,+\infty)$ with infinite logarithmic measure, such that for all $z$ satisfying $|z|=r \in E_{2}$, and $r$ sufficiently large, we have

$$
\begin{gathered}
M\left(r, a_{l}(z)\right)>\exp \left\{\alpha_{2} r^{\rho}\right\}, \text { and } \\
M\left(r, a_{j}(z)\right)<\exp \left\{\alpha_{1} r^{\rho}\right\}, \quad j \in I \backslash\{l\} .
\end{gathered}
$$

If $l \neq n+1$, combining these inequalities (3.1)-(3.5) with (1.1), we get

$$
\begin{aligned}
& \left|a_{l}(z)\right| \\
= & \left|\frac{1}{f\left(q^{l} z\right)}\right|\left|-\sum_{j \in I \backslash\{l, n+1\}} a_{j}(z) f\left(q^{j} z\right)-\sum_{j \in\{0, \ldots, n\} \backslash I} a_{j}(z) f\left(q^{j} z\right)+a_{n+1}(z)\right| \\
\leq & \left.\left|\frac{1}{f\left(q^{l} z\right)}\right|\left|\sum_{j \in I \backslash\{l, n+1\}} M\left(r, a_{j}(z)\right)\right| f\left(q^{j} z\right)\left|+\sum_{j \in\{0, \ldots, n\} \backslash I}\right| a_{j}(z)|| f\left(q^{j} z\right) \mid\right) \\
& +\left|\frac{1}{f\left(q^{l} z\right)}\right|\left|a_{n+1}(z)\right| \\
\leq & (n+1) \exp \left\{\alpha_{1} r^{\rho}+2 r^{\sigma+\varepsilon_{1}}\right\}
\end{aligned}
$$

for all $z=r e^{i \theta}$ satisfying $|z|=r \in E_{2} \backslash E_{1}$, and $M\left(r, a_{l}(z)\right)=\left|a_{l}(z)\right|$. This then implies that

$$
\exp \left\{\left(\alpha_{2}-\alpha_{1}\right) r^{\rho}-2 r^{\sigma+\varepsilon_{1}}\right\} \leq n+1,
$$

holds for sufficiently large $r \in E_{2} \backslash E_{1}$, which is a contradiction.

If $l=n+1$, we can use the same method to deduce a similar contradiction. The first assertion now follows immediately.

We next show that $\tau(f) \geq \tau\left(a_{n+1}\right)-\tau$ when $l=n+1$. Assume contrary to the assertion that $\tau(f)<\tau\left(a_{n+1}\right)-\tau$.

From Lemma 3.1, for any given $\varepsilon_{2}\left(0<4 \varepsilon_{2}<\min \left\{\tau\left(a_{n+1}\right)-\tau-\tau(f), \rho-\tilde{\rho}\right\}\right)$, there exists a set $E_{3} \subset(1,+\infty)$ with finite measure, such that for all $z$ satisfying $|z|=r \notin[0,1] \cup E_{3}$, and $r$ sufficiently large, we have

$$
\left|a_{j}(z)\right| \leq \exp \left\{r^{\tilde{\rho}+\varepsilon_{2}}\right\} \leq \exp \left\{r^{\rho-\varepsilon_{2}}\right\} \quad j \in\{0, \ldots, n\} \backslash I .
$$

By Lemma 3.2 and again the definition of the type of an entire function, we see that there exists a set $E_{4} \subset(1,+\infty)$ with infinite logarithmic measure, such that for all $z=r e^{i \theta}$ satisfying $|z|=r \in E_{4},\left|a_{n+1}\left(r e^{i \theta}\right)\right|=M\left(r, a_{n+1}\right)$ and $r$ sufficiently large, we have

$$
M\left(r, a_{n+1}(z)\right)>\exp \left\{\left(\tau\left(a_{n+1}\right)-\varepsilon_{2}\right) r^{\rho}\right\},
$$




$$
M\left(r, f\left(q^{j} z\right)\right)<\exp \left\{\left(\tau(f)+\varepsilon_{2}\right)\left(|q|^{j} r\right)^{\rho}\right\} \leq \exp \left\{\left(\tau(f)+\varepsilon_{2}\right) r^{\rho}\right\}
$$

for $j=0, \ldots, n$, and

$$
M\left(r, a_{j}(z)\right)<\exp \left\{\left(\tau+\varepsilon_{2}\right) r^{\rho}\right\}, \quad j \in I \backslash\{n+1\} .
$$

Combining inequalities (3.6)-(3.9) with (1.1), we get

$$
\begin{aligned}
\left|a_{n+1}(z)\right| & =\left|\sum_{j \in I \backslash\{n+1\}} a_{j}(z) f\left(q^{j} z\right)+\sum_{j \in\{0, \ldots, n\} \backslash I} a_{j}(z) f\left(q^{j} z\right)\right| \\
& \leq \sum_{j \in I \backslash\{n+1\}} M\left(r, a_{j}(z)\right) M\left(r, f\left(q^{j} z\right)\right)+\sum_{j \in\{0, \ldots, n\} \backslash I}\left|a_{j}(z)\right| M\left(r, f\left(q^{j} z\right)\right) \\
& \leq(n+1) \exp \left\{\left(\tau+\tau(f)+2 \varepsilon_{2}\right) r^{\rho}\right\}
\end{aligned}
$$

for all $z=r e^{i \theta}$ satisfying $|z|=r \in E_{4} \backslash E_{3}$, and $M\left(r, a_{n+1}(z)\right)=\left|a_{n+1}(z)\right|$. This then implies that

$$
\exp \left\{\left[\tau\left(a_{n+1}\right)-\tau-\tau(f)-3 \varepsilon_{2}\right] r^{\rho}\right\} \leq n+1,
$$

holds for sufficiently large $r \in E_{4} \backslash E_{3}$, which is a contradiction. Hence we have proven that $\tau(f) \geq \tau\left(a_{n+1}\right)-\tau$.

\section{The case of $|q|=1$}

In the previous sections, we assumed that $q$ was a non-zero complex constant with $0<|q|<1$. The following mainly deals with the corresponding results if $|q|=1$. Moreover, a further result about the solutions of (1.1) is also given.

Theorem G ([5]). Suppose $a_{0}(z), \ldots, a_{n+1}(z)$ are polynomials and $q$ is a constant with $|q|=1$ and $q \neq 1$. If (1.1) possesses a nonconstant meromorphic solution $f(z)$, then

(i) if $f(z)$ is a rational function of the form

$$
f(z)=\frac{p_{0}+p_{1} z+\cdots+p_{m} z^{m}}{q_{0}+q_{1} z+\cdots+q_{t} z^{t}},
$$

then $t-m \leq \max \left\{\operatorname{deg} a_{j}: j=0, \ldots, n\right\}-\operatorname{deg} a_{n+1}$.

(ii) if there is some $l \in\{0, \ldots, n\}$, such that

$$
\operatorname{deg} a_{l}>\max _{j \in\{0, \ldots, n\} \backslash\{l\}}\left\{\operatorname{deg} a_{j}\right\},
$$

and $f(z)$ is a transcendental function, then $f(z)$ has infinitely many poles.

Now we get the Theorem 4.1 as a continuation of Theorem 5.1 in [13] below.

Theorem 4.1. Suppose the coefficients $a_{0}(z), \ldots, a_{n+1}(z)$ of (1.1) are meromorphic, $a_{0}(z) a_{n}(z) \not \equiv 0$, and $q=e^{i \theta}, \theta=\lambda \pi, \lambda \in(0,2) \backslash \mathbb{Q}$. If (1.1) possesses a nonconstant meromorphic solution $f(z)$, then the non-zero poles of $f(z)$ are of the form $\left\{\frac{z}{q^{k}} \mid a_{0}(z)=0\right.$ or $\left.a_{j}(z)=\infty, j=1, \ldots, n+1, k \in \mathbb{N} \cup\{0\}\right\}$.

From Theorem G and Theorem 4.1, the following consequence obviously holds. 
Corollary 4.2. Suppose the coefficients $a_{0}(z), \ldots, a_{n+1}(z)$ of $(1.1)$ are polynomials, $a_{0}(z) a_{n}(z) \not \equiv 0$, and $q=e^{i \theta}, \theta=\lambda \pi, \lambda \in(0,2) \backslash \mathbb{Q}$. If (1.1) possesses a nonconstant meromorphic solution $f(z)$, then $f(z)$ has at most finitely many non-zero poles, which are of the form $\left\{\frac{z}{q^{k}} \mid a_{0}(z)=0, k \in \mathbb{N} \cup\{0\}\right\}$. Moreover, if there is some $l, 0 \leq l \leq n$, such that

$$
\operatorname{deg} a_{l}>\max _{j \in\{0, \ldots, n\} \backslash\{l\}}\left\{\operatorname{deg} a_{j}\right\},
$$

then $f(z)$ must be a rational function.

We give some examples to show that there exist some equations of the form (1.1), which satisfy Theorem 4.1 or Corollary 4.2 .

Examples. (1) $f(z)=\frac{e^{z}}{(z-1)(q z-1)}$ is a meromorphic function and satisfies the equation

$$
a_{1}(z) f(q z)+a_{0}(z) f(z)=a_{2}(z)
$$

where

$q=e^{i \sqrt{2} \pi}, \quad a_{0}(z)=-(z-1) e^{q z}, \quad a_{1}(z)=\left(q^{2} z-1\right)\left(e^{z}+1\right), \quad a_{2}(z)=\frac{e^{q z}}{q z-1} ;$

(2) $f(z)=\frac{1}{z+1}$ is a rational function and satisfies the equation

$$
a_{1}(z) f(q z)+a_{0}(z) f(z)=a_{2}(z),
$$

where

$$
q=e^{i \sqrt{2} \pi}, \quad a_{0}(z)=z^{2}(z+1), \quad a_{1}(z)=q z+1, \quad a_{2}(z)=z^{2}+1 ;
$$

(3) $f(z)=z+1$ is a polynomial and satisfies the equation

$$
a_{2}(z) f\left(q^{2} z\right)+a_{1}(z) f(q z)+a_{0}(z) f(z)=a_{3}(z)
$$

where

$q=e^{i \sqrt{2} \pi}, \quad a_{0}(z)=1-z, \quad a_{1}(z)=q z-1, \quad a_{2}(z)=z^{2}, \quad a_{3}(z)=q^{2} z^{2}(z+1)$.

In the theorems above, we investigated the poles of meromorphic solutions of (1.1). Now we give a result on the zeros of meromorphic solutions of (1.1).

Theorem 4.3. Suppose the coefficients $a_{0}(z), \ldots, a_{n+1}(z)$ of $(1.1)$ are polynomials, $a_{0}(z) a_{n}(z) \not \equiv 0$, and $q=e^{i \theta}, \theta=\lambda \pi, \lambda \in(0,2) \backslash \mathbb{Q}$. If (1.1) possesses a nonconstant finite order meromorphic solution $f(z)$, then $\lambda(f)=\rho(f)$.

As counterparts of Theorem 1.2, Theorem 1.3 and Corollary 1.4, we give the following results. Proofs of Theorem 4.4 and Theorem 4.5 are similar to the proof of Theorem 1.3 and thus omitted.

Theorem 4.4. Suppose that the coefficients $a_{0}(z), \ldots, a_{n+1}(z)$ in $(1.1)$ are meromorphic and of finite order $\leq \rho$ and $|q|=1$. If for any given $\varepsilon>0$, there exist some $l, 0 \leq l \leq n+1$, and an unbounded domain $D \subset \mathbb{C}$, such that for all $z \in D$,

$$
\left|a_{l}(z)\right| \geq \exp \left\{\alpha r^{\rho-\varepsilon}\right\}
$$


and

$$
\left|a_{j}(z)\right| \leq \exp \left\{\beta r^{\rho-\varepsilon}\right\}, \quad j=0,1, \ldots, l-1, l+1, \ldots, n+1,
$$

where $\alpha>\beta>0$ are positive real numbers, then each non-zero meromorphic solution of $(1.1)$ is of order $\rho(f) \geq \rho$.

Theorem 4.5. Let $|q|=1$ and the coefficients $a_{0}(z), \ldots, a_{n+1}(z)$ in (1.1) be entire and of finite order $\leq \rho$ such that among those coefficients having the maximal order $\rho:=\max _{0 \leq j \leq n+1} \rho\left(a_{j}\right)$, exactly one denoted by $a_{l}(z)$ has its type strictly greater than the others. Then each non-zero entire solution of (1.1) is of order $\rho(f) \geq \rho$. Moreover, if $l=n+1$ and $\rho(f)=\rho$, then we have $\tau(f) \geq \tau\left(a_{n+1}\right)-\max \left\{\tau\left(a_{j}\right): \rho\left(a_{j}\right)=\rho, j \neq n+1\right\}$.

Corollary 4.6. Under the assumptions of Theorem 4.5, if $\tau\left(a_{n+1}\right)=\infty$, then each non-zero entire solution of (1.1) satisfying $\rho(f)=\rho$ is of type $\tau(f)=\infty$.

We recall the following examples given in [5] to show that the growth estimate of meromorphic solutions of (1.1) in Theorems 4.5 is sharp, where examples (1) and (3) show that $\rho(f)>\rho$ may hold in Theorems 4.5. What's more, Example (2) shows that the type estimate is also sharp.

Examples. (1) $e^{z} f(q z)-e^{3 z} f(z)=z e^{4 z}+z, \quad q=-1, f(z)=e^{z^{2}-z}-z e^{z}$;

(2) $e^{z} f(q z)+\left(e^{z}-e^{-z}\right) f(z)=e^{2 z}, \quad q=-1, f(z)=e^{z}$

(3) $e^{z} f\left(q^{2} z\right)+e^{z} f(q z)-\left(e^{z}+e^{-z}\right) f(z)=0, \quad q=-1, f(z)=e^{z^{2}+z}$.

\section{Proof of Theorem 4.1}

Set $B=\left\{z \mid a_{j}(z)=\infty, j=1, \ldots, n+1\right\}$. Suppose that there exists a pole $z_{0}$ of $f(z)$ such that for any $b_{s} \in B, z_{0}$ is not of the form $\frac{b_{s}}{q^{k}}$ (for any $k \in \mathbb{N} \cup\{0\})$. That is

$$
a_{j}\left(q^{k} z_{0}\right) \neq \infty, j=1, \ldots, n+1, \forall k \in \mathbb{N} \cup\{0\} .
$$

As $q=e^{i \theta}, \theta=\lambda \pi, \lambda \in(0,2) \backslash \mathbb{Q}$, we see that for any $k, l \in \mathbb{N} \cup\{0\}, k \neq l$,

$$
(k-l) \theta \not \equiv 0(\bmod 2 \pi) \text {, }
$$

which yields that

$$
q^{k} \neq q^{l}
$$

Since $f(z)$ is a meromorphic function, we see that $f(z)$ has at most finitely many poles on the circle $|z|=\left|z_{0}\right|$. Then $f(z)$ has at most finitely many poles which are of the form $q^{k_{m}} z_{0}, m=0, \ldots, t$. such that $0 \leq k_{0} \leq k_{1} \leq \cdots \leq k_{t}$. That is

$$
f\left(q^{k_{t}} z_{0}\right)=\infty, f\left(q^{k_{t}+j} z_{0}\right) \neq \infty, j=1, \ldots, n+1 .
$$

From (1.1), we have

$$
a_{0}\left(q^{k_{t}} z_{0}\right) f\left(q^{k_{t}} z_{0}\right)=-\sum_{j=1}^{n} a_{j}\left(q^{k_{t}} z_{0}\right) f\left(q^{k_{t}+j} z_{0}\right)+a_{n+1}\left(q^{k_{t}} z_{0}\right) .
$$


Then by (5.1)-(5.3), we conclude that $a_{0}\left(q^{k_{t}} z_{0}\right)=0$, which means that, there exists a point $z^{*}$ such that

$$
a_{0}\left(z^{*}\right)=0, z_{0}=\frac{z^{*}}{q^{k_{t}}} .
$$

Thus Theorem 4.1 is proved.

\section{Proof of Theorem 4.3}

Lemma $6.1([17])$. Let $f_{j}(z)(j=1, \ldots, n, n \geq 2)$ be meromorphic functions, $g_{j}(z)(j=1, \ldots, n)$ be entire functions, and satisfy

(i) $\sum_{j=1}^{n} f_{j}(z) e^{g_{j}(z)} \equiv 0$;

(ii) when $1 \leq j<k \leq n, g_{j}(z)-g_{k}(z)$ is not a constant; and

(iii) when $1 \leq j \leq n, 1 \leq h<k \leq n$,

$$
T\left(r, f_{j}\right)=o\left\{T\left(r, e^{g^{h}-g^{k}}\right)\right\},(r \rightarrow \infty, r \notin E),
$$

where $E \subset(1, \infty)$ is of finite linear measure or finite logarithmic measure. Then $f_{j}(z) \equiv 0(j=1, \ldots, n)$.

Proof of Theorem 4.3. Assume contrary to the assertion that $\lambda(f)<\rho(f)$. By Corollary 4.2 , we see that $f(z)$ has at most finitely many non-zero poles, then $\lambda\left(\frac{1}{f}\right)=0<\rho(f)$. Thus, since $f(z)$ is a meromorphic function of finite order, then we can set

$$
f(z)=P(z) e^{p(z)},
$$

where $P(z)$ is a meromorphic function such that $\rho(P)<\rho(f)$, and $p(z)$ is a polynomial such that $d=\operatorname{deg}(p)=\rho(f)$. Now set

$$
p(z)=\alpha_{d} z^{d}+\alpha_{d-1} z^{d-1}+\cdots+\alpha_{0},
$$

where $\alpha_{d} \neq 0, \alpha_{d-1}, \ldots, \alpha_{0}$ are constants.

Substituting (6.1) into (1.1), we get

$$
\sum_{j=0}^{n} a_{j}(z) P\left(q^{j} z\right) e^{p\left(q^{j} z\right)}-a_{n+1}(z)=0 .
$$

As $q=e^{i \theta}, \theta=\lambda \pi, \lambda \in(0,2) \backslash \mathbb{Q}$, we see that for any $0 \leq k<l \leq n, q^{k} \neq q^{l}$. Clearly,

$$
p\left(q^{k} z\right)-p\left(q^{l} z\right)=\alpha_{d}\left(q^{k d}-q^{l d}\right) z^{d}+\cdots+\alpha_{1}\left(q^{k}-q^{l}\right) z
$$

and hence $\operatorname{deg}\left(p\left(q^{k} z\right)-p\left(q^{l} z\right)\right)=d$.

Since $a_{0}(z), \ldots, a_{n+1}(z)$ of $(1.1)$ are polynomials and by $\rho(P)<d$, we have, for $0 \leq j \leq n, 0 \leq k<l \leq n, 0 \leq h \leq n$,

$$
\begin{aligned}
T\left(r, a_{j}(z) P\left(q^{j} z\right)\right) & =o\left\{T\left(r, e^{p\left(q^{k} z\right)-p\left(q^{l} z\right)}\right)\right\}=o\left\{T\left(r, e^{p\left(q^{h} z\right)}\right)\right\}, \text { and } \\
T\left(r, a_{n+1}(z)\right) & =o\left\{T\left(r, e^{p\left(q^{k} z\right)-p\left(q^{l} z\right)}\right)\right\}=o\left\{T\left(r, e^{p\left(q^{h} z\right)}\right)\right\} .
\end{aligned}
$$


Applying Lemma 6.1 to (6.2) yields

$$
a_{j}(z) \equiv 0, j=0, \ldots, n+1
$$

This is a contradiction. Thus Theorem 4.3 is proved.

Acknowledgements. The authors are grateful to the referee for his/her helpful suggestions. These comments greatly improved the readability of the paper.

\section{References}

[1] M. Ablowitz, R. G. Halburd, and B. Herbst, On the extension of the Painlevé property to difference equations, Nonlinearity 13 (2000), no. 3, 889-905.

[2] W. Bergweiler, K. Ishizaki, and N. Yanagihara, Meromorphic solutions of some functional equations, Methods Appl. Anal. 5 (1998), no. 3, 248-258.

[3] — Growth of meromorphic solutions of some functional equations I, Aequationes Math. 63 (2002), no. 1-2, 140-151.

[4] W. Bergweiler and J. K. Langley, Zeros of differences of meromorphic functions, Math. Proc. Cambridge Philos. Soc. 142 (2007), no. 1, 133-147.

[5] B. Q. Chen, Z. X. Chen, and S. Li, Properties on solutions of some q-difference equations, Acta Math. Sin. (Engl. Ser.) 26 (2010), no. 10, 1877-1886.

[6] Z. X. Chen and K. H. Shon, On zeros and fixed points of differences of meromorphic functions, J. Math. Anal. Appl. 344 (2008), no. 1, 373-383.

[7] Y. M. Chiang and S. J. Feng, On the Nevanlinna characteristic of $f(z+\eta)$ and difference equations in thecomplex plane, Ramanujan J. 16 (2008), no. 1, 105-129.

[8] G. Gundersen, J. Heittokangas, I. Laine, J. Rieppo, and D. G. Yang, Meromorphic solutions of generalized Schröder equations, Aequationes Math. 63 (2002), no. 1-2, 110135.

[9] R. G. Halburd and R. Korhonen, Difference analogue of the lemma on the logarithmic derivative with applications to difference equations, J. Math. Anal. Appl. 314 (2006), no. 2, 477-487.

[10] Nevanlinna theory for the difference operator, Ann. Acad. Sci. Fenn. Math. 31 (2006), no. 2, 463-478.

[11] W. K. Hayman, Meromorphic Functions, Clarendon Press, Oxford, 1964.

[12] Angular value distribution of power series with gaps, Proc. Lond. Math. Soc. (3) $\mathbf{2 4}$ (1972), no. 4, 590-624.

[13] J. Heittokangas, I. Laine, J. Rieppo, and D. G. Yang, Meromorphic solutions of some linear functional equations, Aequationes Math. 60 (2000), no. 1-2, 148-166.

[14] I. Laine, Nevanlinna Theory and Complex Differential Equations, Walter de Gruyter, Berlin, 1993.

[15] S. Saks and A. Zygmund, Analytic Functions, Monografie Mat. (Engl. Transl.) Tom 28, Warsaw, 1952.

[16] J. Tu and C. F. Yi, On the growth of solutions of a class of higher order linear differential equations with coefficients having the same order, J. Math. Anal. Appl. 340 (2008), no.1, 487-497.

[17] C. C. Yang and H. X. Yi, Uniqueness Theory of Meromorphic Functions, Math. Appl., vol. 557, Kluwer Academic Publishers Group. Dordrecht, 2003.

[18] L. Yang, Value Distribution Theory and New Research, Science Press, Beijing, 1982. 
BAOQIN Chen

School of Mathematical Sciences

South China Normal University

Guangzhou, 510631, P. R. China

E-mail address: chenbaoqin_chbq@126.com

ZONGXUAN CHEN

School of Mathematical Sciences

South China Normal University

Guangzhou, 510631, P. R. China

E-mail address: chzx@vip.sina.com 